\title{
Avaliação da compreensão dos pacientes hipertensos a respeito da hipertensão arterial e seu tratamento versus controle pressórico
}

Patients' comprehension of arterial hypertension and its management versus blood pressure control

Marciele V. Guimarães ${ }^{1}$ Luiz Fernando O. Ribas ${ }^{2}$

\section{RESUMO}

Este artigo tem como objetivo avaliar a compreensão dos pacientes hipertensos mediante medidas de saúde preconizadas pela Secretaria Municipal de Curitiba adotadas na Unidade Básica de Saúde Vista Alegre quanto à hipertensão arterial e seu tratamento. Realizou-se coleta de dados sobre ações promovidas no local, entrevista informal com profissionais de saúde sobre o Programa do Hipertenso e questionário a 50 pacientes inscritos no Programa e selecionados por sorteio, com abordagem na Unidade ou domiciliar, sendo mensurada sua pressão arterial. Avaliou-se que: 38\% não sabem definir hipertensão; $72 \%$ apresentam nervosismo/preocupação como causa; $84 \%$ indicam derrame como conseqüência de pressão elevada; a maioria sabe qual é seu medicamento e como ministrá-lo; $41 \%$ esquecem, algumas ou várias vezes, de tomá-los, $10 \%$ pensam não haver problema em deixar de ingeri-lo, às vezes; boa parte dos que não tomam é por esquecimento, e não por dificuldade de acesso à medicação. Além disso, dois terços não seguem dieta favorável e $52 \%$ não praticam atividades físicas, mas quase todos dizem que estas ajudam a controlar a pressão. Dos pacientes, os que já sofreram derrame ou infarto seguem melhor as recomendações. Dos $50,96 \%$ concordam que emagrecer também ajuda, mas $72 \%$ estão acima do peso. Relativo à mensuração, $26 \%$ estavam com pressão considerada normal; $18 \%$ com hipertensão grave e $20 \%$, moderada. Muitos não sabem definir hipertensão, mas, apesar de não modificarem seus hábitos de vida, sabem as conseqüências da doença e como controlá-la. Não se adaptam a alterações dos hábitos, argumentando falta de tempo e estímulo ou evidência de seu real benefício.

PALAVRAS-CHAVE:

- Hipertensão Arterial;

- Terapia;

- Monitores de Pressão Arterial.
KEY-WORDS:

- Hypertension;

- Therapy;

- Blood Pressure Monitors.

${ }^{1}$ Médica Residente, Programa de Medicina de Família e Comunidade, Pontifícia Universidade Católica do Paraná.

2 Professor, Disciplina de Atenção Primária à Saúde, Departamento de CM, Universidade Federal do Paraná. 


\section{ABSTRACT}

This paper aims to evaluate the comprehension of hypertensive patients of their condition and its management through health practices recognized by the Health Secretariat of the city of Curitiba and adopted in the Basic Health Unit "Vista Alegre". The methodology used included collection of data about health actions promoted by the Unit, informal interviews with health professionals about how Hypertension Programs work and interviews based on a questionnaire with 50 patients participating in the Hypertension Program, selected and approached in the Health Unit or in household visits, who had their blood pressure taken after the interview. Thirty eight percent could not define hypertension, $72 \%$ assumed that nervousness or preoccupation were causes of hypertension, $84 \%$ mentioned stroke as consequence of high blood pressure, most of patients knew their medication and how to take it, $41 \%$ admitted forgetting to take their medication occasionally or often, $8 \%$ revealed not to take it correctly, $10 \%$ felt that not taking it sometimes does not make difference. Great part of those not taking their medication declared that this happens because they do not remember to take it, not for lacking it. Two thirds had unfavorable dietary habits and 52\% did not engage in physical exercise but most believed that diet and exercise help controlling high blood pressure. Ninety six percent agreed that loosing weight also helps, but $72 \%$ were ovenweight. Only 13 (26\%) had normal blood pressure; 18\% presented with severe hypertension and $20 \%$ with moderate hypertension. Analysis of the collected data showed that many patients are unable to define hypertension but know how to control it and are aware of its consequences.
The excuses offered for not adapting to changes in their life stile were lack of time, stimulation and proof of benefits.

\section{I - Introdução}

A Hipertensão Arterial Sistêmica (HAS) continua sendo um grande problema de saúde em todo o mundo 1 . Não existem dados populacionais da prevalência da hipertensão no país; com base em dados obtidos em alguns estudos de base populacional, estima-se que afete aproximadamente um quarto da população brasileira ${ }^{2}$. No Brasil, as doenças do aparelho circulatório constituem a principal causa de morte entre idosos, correspondendo a cerca de $40 \%$ dos óbitos desta população. Dentre essas, as doenças cerebrovasculares e as isquêmicas do coração são as duas causas mais freqüentes de óbitos ${ }^{3}$. Sabe-se que a HAS é o principal fator de risco para doenças cerebrovasculares ${ }^{4,5}$, patologia de altas taxas de morbidade, além de apresentar importante associação com doenças isquêmicas do coração e insuficiência cardíaca congestiva ${ }^{6}$, insuficiência renal e doença vascular periférica ${ }^{1}$. A HAS é, portanto, um dos fatores de risco mais preocupantes, pois, na maioria das vezes, não apresenta sintomas ${ }^{7}$, além de ser a causa modificável mais importante na morbidade e mortalidade cardiovasculares ${ }^{8,3}$. Trata-se de uma afecção geralmente de curso silencioso, de alta prevalência na população adulta, acometendo esta população cada vez mais precocemente. Embora seu diagnóstico e tratamento parecerem simples, permanecem de difícil manejo, representando um desafio aos sistemas de saúde ${ }^{9}$. Mesmo em países desenvolvidos como o Canadá, poucos, 
em torno de $16 \%$, são os que alcançam adequado controle da pressão arterial ${ }^{1}$.

O diagnóstico da HAS é feito quando indivíduos a partir de 18 anos, não utilizando medicação anti-hipertensiva e sem comorbidade, apresentam persistência de pressão arterial sistólica (PAS) igual ou maior a $140 \mathrm{mmHg}$ e/ou pressão arterial diastólica (PAD) igual ou maior a $90 \mathrm{mmHg}$. Esse diagnóstico é confirmado em pelo menos duas ou mais leituras, feitas em três ou mais avaliações de saúde, com intervalos regulares $^{9}$. Conforme as recomendações canadenses para manejo de hipertensão ${ }^{10}$, a abordagem terapêutica da HAS sem comorbidades ou lesão de órgão alvo objetiva manter a pressão arterial abaixo dos níveis citados; quando há associação com diabetes mellitus ou doença renal crônica, o alvo pressórico é $P A S<130 \mathrm{mmHg}$ e $P A D<80 \mathrm{mmHg}$; e com proteinúria $>1 \mathrm{~g} / \mathrm{dia}, \mathrm{PAS}<125 \mathrm{mmHg}$ e $\mathrm{PAD}<75 \mathrm{mmHg}$

É bem conhecido que os medicamentos anti-hipertensivos reduzem a pressão arterial (PA) e a mortalidade relacionada à $\mathrm{HAS}^{5,11}$. Porém, em poucos casos o uso do medicamento é feito de maneira correta, o que é um problema inerente do tratamento de condições crônicas e assintomáticas, sendo um dos motivos da baixa efetividade do tratamento medicamentoso da HAS. A baixa aderência à medicação pode também contribuir para a resistência aparente à terapia em mais de um terço dos pacientes ${ }^{11}$.

Poucos são os pacientes que atingem os níveis pressóricos estabelecidos pelos critérios da Organização Mundial da Saúde (OMS). Esta confirma, em seu boletim, que os níveis de compreensão da doença, de tratamento e de controle da HA são baixos ${ }^{12}$. De acordo com estudos prévios $^{3,12}$, as mulheres apresentam maior probabilidade de estar em tratamento e de ter a sua PA melhor controlada, além de saberem ser hipertensas. O uso de serviços de saúde aumenta a chance de conhecimento da condição de hipertenso, ao aumentar a probabilidade de diagnóstico ${ }^{3}$. Uma visita ao médico no ano anterior, aquisição educacional mais alta e o sexo feminino emergiram como correlatos importantes do conhecimento da hipertensão, de acordo com o boletim da OMS ${ }^{12}$.

A análise sobre HAS no Rio Grande do Sul ${ }^{13}$ evidenciou que seu descontrole é alarmante, pois em torno de $80 \%$ dos indivíduos que sabiam ser hipertensos possuíam PA>140/90mmHg, e, daqueles sob medicação anti-hipertensiva, apenas $25,6 \%$ obtiveram controle da PA, mostrando que o controle efetivo da doença não tem alcançado nível satisfatório, representando um desafio a ser encarado pelo sistema de saúde.

Resultados preliminares da Pesquisa Corações do Brasil ${ }^{7}$ mostraram: $93,5 \%$ da população hipertensa e em tratamento com algum antihipertensivo não atingiu nível pressórico considerado ótimo; a falta de cuidado com a saúde é mais presente entre os homens que entre as mulheres. Dos 6,5\% que se tratam e atingiram a meta, $65 \%$ são mulheres; $48,1 \%$ da população com pressão alta sabe do problema e não faz qualquer tipo de tratamento, sendo que $60 \%$ dos homens não se cuidam, enquanto entre as mulheres esse índice é de 40\%; o tratamento da hipertensão não depende do rendimento familiar. No grupo que recebe menos de 10 salários mínimos por mês, o índice é de $49 \%$ de adesão, enquanto no que re- 
cebe de 1 a 5 salários, a porcentagem é bastante semelhante, $50 \%$.

Pesquisa em amostra significativa da população espanhola em 1998 também demonstrou que somente pequena parcela $(15,5 \%)$ daqueles sob tratamento e que sabiam de sua condição de hipertensos tinha sua PA controlada, constituindo $5 \%$ dos hipertensos ${ }^{14}$. Os resultados foram semelhantes em Minessota ${ }^{15}$, onde apenas 16,6\% dos hipertensos sabiam de sua doença, estavam tratados e com controle pressórico. Também em amostra significativa entre os chineses ${ }^{8}$, a HAS controlada foi mais comum entre pessoas cuja PA fora medida de um a cinco anos antes da visita do estudo e, entre os que conheciam seu diagnóstico de hipertensão, aqueles submetidos a modificação do estilo de vida. Outro estudo na China ${ }^{16}$ revelou que mulheres sabiam mais da doença e tinham melhor controle pressórico; apenas 33,8\% delas estavam sendo tratadas, sendo que $6,1 \%$ dos homens e $8,3 \%$ das mulheres atingiram controle da PA.

Firmo et al. ${ }^{17}$ observaram, por meio de entrevista, que hipertensos pensam que a pressão alta é desencadeada especialmente por problemas familiares e pode ser facilmente reconhecida pela identificação de algumas manifestações específicas, associando-se explicitamente ao aparecimento de alguns problemas como tontura, mal-estar, cefaléia, aumento da pressão. Eles também acreditam que o quadro só é considerado problemático quando a pressão sobe, sendo o único momento em que as intervenções são vistas como necessárias. Dessa forma, a importância de buscar tratamento ou seguir corretamente a prescrição médica fica na dependência da identificação de um aumento da pressão, seja pela presença de sintomas específicos ou de condições subjetivas favoráveis à elevação da pressão.

O nível de escolaridade, segundo Góes et al. ${ }^{18}$, tem influência no grau de compreensão. Além disso, Simão et al. cita que o grau de instrução está relacionado à compreensão do indivíduo acerca do que é uma doença crônica, dos fatores de risco contribuintes para sua instalação e do significado atribuído às orientações sobre alimentação, hábitos de vida e seus efeitos sobre a saúde ${ }^{19}$.

As recomendações de estilo de vida para hipertensão ${ }^{10}$ incluem: abstinência do tabagismo; perder peso, incluindo a educação dietética, aumento de atividade física e modificação do comportamento, sendo que a PA pode ser reduzida em $1.6 / 1.1 \mathrm{mmHg}$ para cada $1 \mathrm{~kg}$ de peso perdido; dieta que enfatize frutas frescas, vegetais $e$ produtos com pouca gordura (em hipertensos, essa dieta reduz a PA em 11,4/5,5mmHg); limitar a ingestão de sódio a $65-100 \mathrm{mmol} / \mathrm{dia}$; fazer de 30 a 60 minutos de exercício dinâmico de moderada intensidade, como caminhada, bicicleta e natação não-competitiva, de quatro a sete dias por semana, limitar o consumo de álcool a duas doses por dia, homens, menos de 14 doses por semana e mulheres, menos de 9 doses por semana; controlar o stress, com intervenções de comportamento cognitivo individualizado e técnicas de relaxamento.

Em revisão realizada por Araújo $^{20}$, foi consenso o benefício trazido pela atividade física, desde que regular, tanto por um componente agudo tardio como pelo efeito crônico da repetição periódi- 
ca e freqüente do exercício físico. Estudo de Martinson et al. ${ }^{21}$ concluiu que, em adultos com doenças crônicas, foi observada mortalidade mais elevada nos fisicamente inativos em um período de 14 meses. Se a inatividade física aponta um risco independente de mortalidade, esforços para manter atividade física em tais pacientes podem trazer benefícios clínicos significantes em curto período.

O consumo regular de uma dieta nutricionalmente completa oferece múltiplos benefícios clínicos na redução do risco de doenças cardiovasculares, em pessoas com risco elevado e do peso corporal ${ }^{22}$. A restrição de sal é também promovida como alvo não-farmacológico efetivo para abordagem na hipertensão leve, assim como importante adjuvante no tratamento farmacológico em hipertensão moderada e grave. Artigo de Ohta ${ }^{23}$ sugere não haver restrição da ingestão de sal mesmo em indivíduos conscientes com relação a essa substância. Na revisão de Fodor et al. ${ }^{24}$ de artigos indexados na base Medline, realizados entre 1966 e 1996, recomenda-se restrição moderada na ingestão de sal a uma média de 90-130mmol/dia (correspondente a 3$7 \mathrm{~g}$ de sal/dia), para hipertensos acima de 44 anos de idade, não sendo recomendada à população normotensa.

O grau de conhecimento e controle da HAS parece ser afetado por fatores sociodemográficos, e a maioria dos hipertensos não tem sua hipertensão controlada 5 . Outro autor refere não haver disparidades quanto a conhecer sua condição de hipertenso dentre diferentes níveis socioeconômicos, mas que o controle da PA é mais pobre nos de categorias ocupacionais de renda inferior, nos que consomem mais bebidas alcoólicas e nos que apresentam níveis inferiores de educação, sendo este ainda relacionado com maior prevalência da doença ${ }^{25}$.

Em comunidades de baixo nível de renda, não se obedece às orientações, mesmo quando essas são conhecidas. Em relação às mudanças no estilo de vida, para adotarem um mais saudável, essas pessoas têm a justificativa de que o abandono de um dos hábitos prejudiciais pode significar a perda de um prazer, o que é relevante a elas em um contexto de vida no qual as oportunidades de satisfação são escassas ${ }^{25}$.

\section{II - Materiais e Métodos}

O presente estudo foi iniciado por entrevistas semi-estrutruradas, com perguntas abertas a profissionais de saúde da Unidade Básica de Saúde (UBS) Vista Alegre, de Curitiba, visando a obter dados sobre o funcionamento do Programa do Hipertenso. Participaram: uma médica, uma enfermeira, duas auxiliares de enfermagem e alguns agentes comunitários de saúde, que descreveram o Programa e ofereceram suas opiniões a respeito do mesmo.

Foi fornecido pela Autoridade Sanitária Local relatório de inscritos no Programa do Hipertenso da UBS Vista Alegre, de 07 de julho de 2005, obtido por meio do Prontuário Eletrônico utilizado pelas Unidades de Saúde de Curitiba.

Foram abordados, portanto, pacientes com diagnóstico de hipertensão inscritos no Programa. Nesta fase, a população do estudo constituiu uma amostra de 50 pacientes, com idade de 28 a 78 anos, que freqüentaram consultas naquela UBS, compareceram à reunião do Programa ou rece- 
beram consultas domiciliares, no período entre 23 de agosto e 05 de setembro de 2005. A seleção foi realizada por sorteio dentre os presentes na listagem de inscritos. Vários selecionados, porém, pertenciam somente ao Programa de Diabetes ou não foram encontrados em casa, sendo substituídos por outros usuários, alguns indicados pelos Agentes Comunitários de Saúde e outros que se encontravam na Unidade.

A respeito do acompanhamento farmacológico, os pacientes foram solicitados a responder sobre o tratamento da hipertensão com remédios e há quanto tempo, quais medicamentos e como foram tomados, se fizeram uso dos remédios corretamente, se achavam que era relevante deixar de tomá-los às vezes, a freqüência e o motivo de não tomá-los.

Para o tratamento não-farmacológico foram abordados temas como a dieta (se ela ajuda a controlar a pressão, se o paciente segue a dieta recomendada e do que se alimenta geralmente), o exercício (se ajuda, se o paciente pratica e, no caso afirmativo, qual, com que freqüência e por quanto tempo ele o pratica) e o peso (se emagrecer pode melhorar a pressão).

Foram realizadas entrevistas individuais com base em questionário, elaborado pelo pesquisador e pré-testado, composto de 34 perguntas objetivas e discursivas, incluindo: 1) identificação; 2) conhecimento geral da doença; 3) características do paciente; 4) tratamento farmacológico; 5) tratamento não-farmacológico e 6) outras informações.

Sobre conhecimento geral da doença, foram feitas as seguintes questões: 1) O que é hipertensão arterial? 2) O que faz a pressão arterial aumentar? 3) O que a pressão arterial alta pode causar? 4) Qual você acha correto? a) somente o remédio controla a pressão arterial ou b) existem outras maneiras de controlar a pressão, além do remédio? Quais? Quanto às características do paciente, foram feitas perguntas sobre o cumprimento do que foi ensinado para cuidar da hipertensão, há quanto tempo haviam descoberto a doença, quantas consultas tinham feito por ano, se já tinham participado das reuniões do programa e se estas tinham sido proveitosas.

Foram obtidas também outras informações como: o fato de saber se e quando a pressão está controlada; as conseqüências do tabagismo; ter apresentado doenças como AVC, IAM, trombose, ICC ou insuficiência renal; possuir diabete, cardiopatia, doença renal, depressão, câncer ou outras enfermidades; achar que seu tratamento antihipertensivo está ou não funcionando.

A entrevista foi precedida da leitura do termo de consentimento livre e esclarecido, elaborado conforme indicação da Resolução 196/96 do Conselho Nacional de Saúde (CNS), cumprindo os princípios éticos contidos na Declaração de Helsinki. A PA foi verificada ao final da entrevista, de modo a garantir o repouso necessário ao procedimento. As medidas foram tomadas no membro superior direito do paciente, sentado com o braço ao nível do coração; a identificação dos valores da PA foi realizada com utilização de um aparelho esfigmomanômetro WelchAllyn-adulto e um estetoscópio Lythmann Classicll. Por fim, foram anotados peso e altura para cálculo do índice de massa corporal e, além disso, foi investigado prontuário eletrônico dos pacientes entrevistados para comparar informações de anti-hipertensivos pres- 
critos, bem como peso e altura.

O projeto deste estudo foi aprovado pelo Comitê de Ética e Pesquisa da Secretaria Municipal de Saúde de Curitiba.

\section{III - Resultados}

\section{III.1 - Observações administrativas}

A UBS Vista Alegre é uma unidade com equipe de saúde da família e possui 489 pacientes ativos (146 homens e 343 mulheres) e 234 inativos (85 homens e 149 mulheres) inscritos no Programa do Hipertenso.

Notou-se, durante visitas domiciliares realizadas para coleta de dados, que raras visitas domiciliares são praticadas por médicos, a despeito de ser uma Unidade de Saúde da Família. Além disso, os nomes de pacientes cadastrados em Programa de Hipertenso e de Diabetes se encontram misturados no mesmo relatório, sem ordem alfabética ou por subárea, o que trouxe dificuldade para a seleção dos usuários participantes. Ademais, existem pacientes inclusos no programa sem critério e outros que não vivem mais na área de abrangência, mas os Agentes Comunitários de Saúde não estão conseguindo descadastrá-los e ficam responsáveis por mais hipertensos que o existente na área de atuação.

Avaliando prontuários eletrônicos, houve dificuldade para se obter dados sobre a medicação atual completa dos pacientes, pois só se pôde verificar a prescrição registrada e os medicamentos fornecidos, mas não as prescrições escritas à mão ou por outros profissionais fora da rede integrada ao Prontuário Eletrônico. Peso e altura não foram encontrados em vários prontuários.
Para os dias de reunião de sábado, há insa- tisfação da parte dos pacientes, que não se sen- tem bem atendidos, e da parte dos funcionários, que sentem não oferecer atendimento e atenção mais aprimorada pelo tempo insuficiente e pela alta demanda.

\section{III.2 - Entrevistas}

Contatando profissionais da saúde que trabalham na UBS Vista Alegre, localizada no bairro Pilarzinho de Curitiba, notou-se substancial preocupação quanto à aderência dos pacientes hipertensos ao seu tratamento, gerando motivação para realização deste trabalho de pesquisa.

Conforme entrevista com uma médica local, até o momento inexiste maneira de avaliar o entendimento da doença HAS entre os pacientes constantes no programa de hipertensos dessa UBS. Ela acrescenta que, embora seja explicado várias vezes o motivo da necessidade do tratamento, sua forma de administração e o que a falta do tratamento pode proporcionar, diversos pacientes retornam a outras consultas sem praticar o que Ihes foi explicado. Parecem não compreender sua doença e seu tratamento e não mantêm o tratamento ou fazem-no de maneira irregular.

Vários hipertensos, segundo a enfermeira responsável pelo Programa, garantem estar seguindo adequadamente o tratamento, apesar de permanecerem sem controle pressórico, serem resistentes a realizar as orientações prestadas, ou ainda referirem não usar regularmente a medicação.

As ações de saúde empregadas no Programa compreendem: consultas médicas a- 
nuais ou trimestrais, segundo o protocolo, as quais incluem explicações e orientações condizentes com a HAS; orientações e mensurações de pressão arterial pela enfermagem; reuniões e palestras realizadas no segundo e no quarto sábado de cada mês, dias nos quais também é fornecida a medicação anti-hipertensiva, fora da rotina de consulta e passeios trimestrais, estes mais destinados à confraternização entre os idosos.

Com relação às reuniões e palestras realizadas na UBS, observou-se não haver tantos participantes, quanto era esperado. $E$ daqueles que comparecem à UBS no dia da reunião, parte não permanece para participar da palestra, indo embora logo após receber a medicação. De 62 pacientes que assinaram presença em uma reunião, havia 40 assistindo à palestra. A médica esclareceu que a presença nas reuniões é maior entre os mais disciplinados e conscientes em relação ao tratamento. É bem mais difícil encontrar pacientes de menor aderência tanto farmacológica quanto não-farmacológica em suas palestras.

Outros profissionais da UBS comentaram não parecer que realmente são fornecidas todas as informações necessárias para o autocontrole dos pacientes, por motivo da dificuldade em atender à demanda de consultas e de orientações da enfermagem em tempo hábil. Além disso, parece que alguns pacientes não tomam corretamente os anti-hipertensivos por dificuldade visual ou por serem analfabetos, e outros não aceitam bem as orientações individuais, apresentando grande ansiedade, provavelmente devido ao tempo de espera por atendimento. Informam, também, haver necessidade de maior número de funcionários para conseguir oferecer melhor atenção aos pacientes. Nas consultas de enfermagem, precisam reforçar orientação sobre dieta, horário de alimentação, exercícios e importância de tomar a medicação, sendo poucos para orientar média de 60 usuários enquanto entregam medicamentos em cada sábado. Porém, há esperança de melhora no fluxo de atendimento, ao serem passadas as reuniões que ocorriam em apenas dois sábados no mês para dias durante a semana, com pacientes divididos em oito grupos.

\section{III.3 - Questionários aplicados aos hipertensos}

Responderam ao questionário 50 pacientes inscritos e ativos no Programa, com média de idade de 59,7 anos, sendo 41 (82\%) mulheres e nove (18\%) homens. Dentre o grupo, $50 \%$ referiram ser do lar e $13 \%$, aposentados; com relação à escolaridade, $62 \%$ disseram ter estudado até a $4^{\mathrm{a}}$ série do primeiro grau ( $14 \%$ de todos eram analfabetos); $42 \%$ relataram saber que eram hipertensos há mais de 10 anos; 35\% estão em tratamento farmacológico há mais de dez anos; quase todos (98\%) estão sob tratamento medicamentoso e $74 \%$ fazem de duas a quatro consultas médicas por ano.

Quando questionados sobre o que é hipertensão arterial, 38\% não souberam responder e $42 \%$ definiram ser pressão alta; $14 \%$ responderam ser algo relacionado a problemas no sangue; $8 \%$, a cardiopatias; $6 \%$, a nervosismo e $6 \%$, a vasculopatias.

Os fatores mais mencionados como responsáveis por elevação de pressão arterial foram: nervosismo, $72 \%$ dos entrevistados, ingestão de comidas salgadas, $62 \%$; consumo de bebida al- 
coólica, $24 \%$; ingestão de comida gordurosa, $20 \%$; hábito de fumar, $16 \%$; obesidade, $10 \%$; hábito de comer em excesso, $10 \%$ e sedentarismo, $6 \%$. Ainda foram relacionados outros elementos, como depressão, menopausa, calor, hereditariedade, outros alimentos, sono e problemas de rim e coração.

Quando a PA está elevada, 84\% disseram que pode ocorrer derrame (AVC); 54\% citaram infarto (IAM) ou parada cardíaca; $20 \%$, morte; $10 \%$, tontura; $10 \%$, problemas cardíacos; $8 \%$, paralisia, também relacionado a AVC, e alguns ofereceram outras respostas.

Ao serem indagados sobre como controlar a PA, $90 \%$ concordaram haver outras maneiras além de tomar o remédio; os outros $10 \%$ disseram não haver mais nada, sendo necessário somente tomar o remédio para obter controle pressórico. Estes últimos possuíam escolaridade até $4^{\mathrm{a}}$ série e podem não ter compreendido bem a questão, pois, quando questionados sobre dieta e exercício, todos foram favoráveis ao benefício que possuem no controle da PA.

Dos que concordaram haver outras maneiras de controle, deram como resposta: dieta controlada e exercício físico, $46 \%$; não comer ou reduzir o sal, 28\%; não ficar nervoso ou acalmarse, $28 \%$; tomar chá, $10 \%$; não consumir bebida alcoólica, $10 \%$; não fumar, $10 \%$.

Passando-se às questões objetivas, $8 \%$ do total de pacientes referiram não seguir o recomendado para cuidar de sua hipertensão (mas destes, todos freqüentaram reuniões do hipertenso) e $22 \%$ nunca participaram das ações educativas realizadas pelo Programa. Quanto à utilidade das reuniões, $8 \%$ dos participantes dis- seram não valer a pena, justificando não haver muita novidade nas palestras ou haver reuniões em muitos dias do Programa, ou ainda pela desorganização em que se encontrava a UBS nos dias do Programa.

Dos 49 que faziam uso de tratamento farmacológico, quatro (8\%) não souberam dizer quais eram os medicamentos e apenas dois (dentre esses mesmos quatro) não sabiam também como tomá-los; $92 \%$ confirmaram tomálos corretamente; porém, $10 \%$ responderam não haver problema caso deixassem de tomá-los algumas vezes. Levantando-se as respostas e prescrições presentes em prontuário eletrônico: $61 \%$ estão em uso de Captopril, 47\%, de Hidroclorotiazida; $27 \%$, de Nifedipina; $28 \%$, de Propranolol; 6\%, de Furosemida e $15 \%$, de outros. No que diz respeito ao uso do medicamento, $59 \%$ disseram nunca esquecer, $21 \%$ esquecem raramente, $14 \%$ algumas vezes e $6 \%$ várias vezes. Quando não tomam, o fazem principalmente por esquecimento ( $37 \%$ das respostas); $4 \%$ porque se sentem mal; $2 \%$ têm dificuldade de pegar o remédio; $49 \%$ garantem sempre tomar; $8 \%$ deram outros motivos.

Quanto ao tratamento não-farmacológico da hipertensão, 98\% dos pacientes concordam que a dieta ajuda a controlar a pressão; porém, $22 \%$ admitem não seguir a dieta recomendada, sendo que menos de um terço dos entrevistados apresentam dieta considerada favorável, evitando gorduras, doces e massas em excesso e optando por verduras, legumes, frutas e carnes magras.

Quando questionados sobre exercícios físicos, também a grande maioria (96\%) afirma ajudarem no controle da pressão, mas, nesse caso, 
os resultados são ainda piores: $52 \%$ responderam não praticar nenhuma atividade física; das 24 pessoas que a pratica, $22(92 \%)$ citaram a caminhada como atividade regular $(83 \%$ destes por mais 30 minutos), $42 \%$ fazem cinco ou mais vezes na semana e $37 \%$, de três a quatro vezes por semana. Somente dois entrevistados negaram o benefício do fato de emagrecer na melhora da PA. O cálculo do índice de massa corporal (IMC, em $\mathrm{kg} / \mathrm{m}^{2}$ ) apresentou os seguintes resultados: menos de um terço (28\%) está com peso normal (IMC 18,5-24,9), 38\% se encontram em sobrepeso (IMC 25-29,9), 30\% têm obesidade média (IMC 3034,9 ) e $4 \%$ obesidade severa (IMC 35-39,9).

Quanto ao hábito do tabagismo, metade dos entrevistados nunca havia fumado, $42 \%$ são ex-fumantes ou fumantes passivos e $8 \%$ fumam até dez cigarros por dia. Há história de Insuficiência Cardíaca em $16 \%$, de IAM em 10\%, de AVC em $6 \%$ e de Insuficiência Renal em $6 \%$; atualmente, $30 \%$ têm diabete, $26 \%$ possuem cardiopatias (principalmente coronariopatias), 16\% também apresentam depressão.

Quando questionados se a pressão estava controlada ou "boa", 22\% responderam não, e à pergunta "Acha que seu tratamento para hipertensão está funcionando?", 96\% disseram sim. Todavia, após cada entrevista, foram tomadas medidas de pressão arterial, e 74\% estavam com PA acima da faixa tida como normal ${ }^{9}$, considerando-se também os limites pressóricos recomendados para diabetes mellitus, lembrando que $14 \%$ ainda não haviam tomado seus antihipertensivos até o momento da mensuração. De acordo com os níveis pressóricos, $18 \%$ estavam com hipertensão grave (PAS $>=180 \mathrm{mmHg}$ e/ou
PAD> $=110 \mathrm{mmHg}$ ) e $20 \%$ com hipertensão moderada (PAS 160-179mmHg e/ou PAD 100$109 \mathrm{mmHg}$ ).

Não parecem ser claras aos pacientes a definição nem as causas da HAS. Muitos não fazem idéia do que seja ou de que porção do organismo acomete; ao menos, $42 \%$ dizem ser pressão alta, resposta que se pode considerar adequada. Associam muito seu surgimento a situações emocionais, como nervosismo ou preocupações em excesso, e entendem que precisam conseguir ficar calmos e não se incomodar com os problemas que geralmente são dos outros, para assim não ficarem doentes. Aproximadamente dois terços acreditam que comida salgada eleva a pressão e também há várias correlações de hábitos pessoais com o aumento da PA. O AVC é a conseqüência mais comentada (84\%), seguida do IAM e PCR (54\%), sinal de terem noção de morbidades graves serem desencadeadas pela hipertensão arterial. Praticamente não relacionaram HAS a insuficiência renal, insuficiência cardíaca, outras cardiopatias ou trombose.

\section{V - Discussão}

A definição e as causas da HAS não são bem claras aos pacientes, os quais associam muito seu surgimento com as situações emocionais. Dois terços acreditam que sal e hábitos pessoais elevam a PA; o AVC é a conseqüência mais comentada, seguida do IAM e PCR, sinal de terem noção de que morbidades graves podem ser ocasionadas pela HAS. A maioria dos pacientes possui orientação sobre o tratamento farmacológico e não-farmacológico da 
HAS, porém muitos se limitam a obedecer ao uso que pensam ser correto das drogas anti-hipertensivas e não seguem dieta adequada e não praticam nenhum tipo de atividade física. Poucos são os que não conhecem sua medicação nem sabem como usá-la e também que admitem não tomar os remédios corretamente. Dos que praticam exercícios físicos, a maioria o faz na medida adequada (83\% por mais de 30 minutos e $79 \%$ pelo menos três vezes por semana), mas esse número de pessoas ainda é pequeno; os demais oferecem diversas desculpas, como dores articulares, falta de tempo ou de estímulo para manter uma atividade física. Apesar dos esforços, três quartos se mantêm hipertensos, sendo vários com níveis pressóricos bastante elevados.

Espera-se melhora dos resultados com a distribuição das reuniões em grupos menores, em dias separados.

O trabalho de ACS, enfermeiros e médicos é árduo e complexo para conduzir o Programa do Hipertenso, porém, o objetivo do controle pressórico ainda não foi alcançado e medidas visando a uma maior efetividade devem ser empregadas. Discussões democráticas, e não somente unilaterais ou impostas hierarquicamente, devem ser estabelecidas com o verdadeiro fim de proporcionar soluções consistentes àquela comunidade. Certamente há muitas idéias e opiniões cuja expressão se torna limitada ao enfrentar posições hierárquicas em um grupo de funcionários.

Permanece a hipótese a qual os pacientes sabem o que fazer para controlar sua PA, mas não conseguem por em prática as orientações que Ihes são dadas. Há pacientes que não compreen- dem como sua pressão nunca normaliza, tomando tantos remédios e sofrendo tantas restrições alimentares.

As declarações dos profissionais sugerem que pacientes não percebem a real necessidade do tratamento e que efeitos o descontrole da PA poderia provocar, possivelmente por não compreenderem as informações a eles passadas por meio das consultas, reuniões do Programa ou orientações da enfermagem.

\section{V - Referências}

1. Feldman RD, Campbell N, Larochelle $P$, Bolli P, Burgess ED, Carruthers SG. et al. Canadian recommendations for the management of hypertension. Can Med Assoc J. Dec. 14, 1999; 161 (12).

2. Brasil. Ministério da Saúde. Hipertensão arterial. In: Brasil. Ministério da Saúde. Inquérito Domiciliar sobre Comportamentos de Risco e Morbidade Referida de Doenças e Agravos não Transmissíveis: Brasil, 15 capitais e Distrito Federal 2002-2003. Brasília (DF): Coordenação Geral de Agravos e Doenças não Transmissíveis, Secretaria de Vigilância em Saúde; [s.d.]. p.133140.

3. Firmo JOA, Uchoa E, Lima-Costa MF. The Bambui Health and Aging Study (BHAS): factors associated with awareness of hypertension among older adults. Cad. Saúde Pública. mar.-abr. 2004; 20(2): 512-521.

4. Damiani IT, Yokoo El. AVC - acidente vascular cerebral. Informativo do Hospital Tachinni. Rio Grande do Sul: TRB PHARMA; 1995.

5. Rossum CTM, Mheen $\mathrm{H}$, Witteman JCM. Prevalence, Treatment, and Control of Hypertension 
by Sociodemographic Factors Among the Dutch Elderly. Hypertension. 2000; 35: 814.

6. Lima MT, Bucher JSNF, Lima JWO. High blood pressure from the perspective of a lowincome population: an exploratory study of knowledge, attitudes, and practices. Cad. Saúde Pública. jul.-ago. 2004; 20(4): 1079-1087.

7. Sociedade Brasileira de Cardiologia [Notícias]. Pesquisa corações do Brasil revela que $93,5 \%$ da população brasileira hipertensa em tratamento não atinge o nível de pressão arterial considerado ideal. São Paulo: Assessoria de Imprensa da SBC, 26 abr. 2005.

8. Muntner $P$ et al. Factors Associated With Hypertension Awareness, Treatment, and Control in a Representative Sample of the Chinese Population. Hypertension. 2004; 43: 578.

9. Cavalcanti AM, Soares MS, Castellano Junior FF, Sobania SRF. Hipertensão: protocolo de atenção à hipertensão arterial sistêmica. Curitiba (PR): Secretaria Municipal de Saúde; 2004.

10. Canadian Hypertension Education Program. Reference card - 2005 Canadian recommendations for the management of hypertension. Hypertension recommendations. [s.I.:s.n.]; 2005.

11. Bovet $P$ et al. Monitoring one-year compliance to antihypertension medication in the Seychelles. Bull World Health Organ. 2002; 80(1): 33-39.

12. Hypertension Study Group. Prevalence, awareness, treatment and control of hypertension among the elderly in Bangladesh and India: a multicentre study. Bull World Health Organ. 2001; 79(6): 490-500.

13. Gus I, Harzheim E, Zaslavsky C, Medina
C, GUS M. Prevalence, awareness, and control of systemic arterial hypertension in the state of Rio Grande do Sul. Arq. Bras. Cardiol. nov. 2004; 83(5): 424-428.

14. Banegas JR, Rodríguez-Artalejo F, Troca JJC, Guallar-Castillón P, Calero JR. Blood pressure in Spain: distribution, awareness, control, and benefits of a reduction in average pressure. Hypertension. 1998; 32: 998-1002.

15. Meissner I, Whisnant JP, Sheps SG, Schwartz GL, O'Fallon WM, Covalt JL, et al. Detection and control of high blood pressure in the comunity: do we need a wake up call? Hypertension. 1999; 34: 466-471.

16. Dongfeng $G$ et al. Prevalence, Awareness, Treatment, and Control of Hypertension in China. Hypertension. 2002; 40: 920.

17. Firmo JOA, Lima-Costa MF, Uchoa E. The Bambui Health and Aging Study (BHAS): ways of thinking and acting among hypertensive older adults. Cad. Saúde Pública. Jul.-ago. 2004; 20(4): 1029-1040.

18. Goes ELA, Marcon SS. A convivência com a hipertensão arterial. Acta Scientiarum. 2002; 24(3): 819-829.

19. Simão $M$, Nogueira MS, Hayashida $M$, Cesarino EJ. Doenças cardiovasculares: perfil de trabalhadores do sexo masculino de uma destilaria do interior paulista. Rev. Eletrônica de Enfermagem. 2002; 4(2): 27-35.

20. Araújo CGS. Fisiologia do exercício físico e hipertensão arterial: uma breve introdução. Rev Hipertensão. 2001; 4(3).

21. Martinson BC, O'Connor PJ, Pronk NP. Physical inactivity and short-term all-cause 
mortality in adults with chronic disease. Arch Intern Med. Aug. 13-27. 2001; 161(15):1848.

22. McCarron DA, Reusser ME. Reducing Cardiovascular Disease Risk with Diet. Obesity Research. 2001; 9: S335-S340.

23. Ohta $Y$ et al. Relationship between the awareness of salt restriction and the actual salt intake in hypertensive patients. Hypertens Res. 2004; 27(4).

24. Campbell NRC, Burgess E, Taylor G, Wilson E, Cléroux J, Fodor JG. et al. Lifestyle changes to prevent and control hypertension. 5 . Recommendations on dietary salt. Canadian Hypertension Society, Canadian Coalition for High Blood Pressure Prevention and Control, Laboratory Centre for Disease Control at Health Canada, Heart and Stroke Foundation of Canada. CMAJ. 1999. May 4; 160(9 Suppl):S29-34.

25. Gaudemaris R, Lang T, Chatellier G, Larabi L, Lauwers-Cancès V, Maître A, et al. Socioeconomic Inequalities in Hypertension Prevalence and Care: The IHPAF Study. Hypertension. 2002; 39: 1119.

Endereço para correspondência:

Marciele V. Guimarães

Rua Brigadeiro Franco, 2507 ap1703 Rebouças

CEP: 80250-030 - Curitiba - PR

Endereço Eletrônico:

marcielevg@yahoo.com.br 